09

\title{
Лазерное возбуждение крутильных колебаний волоконных микросветоводов
}

\author{
(C) Ф.А. Егоров, В.Т. Потапов \\ Фрязинский фрилиал Института радиотехники и электроники им. В.А.Котельникова РАН, \\ Фрязино, Московская обл., Россия \\ E-mail: egorov-fedor@mail.ru
}

Поступило в Редакцию 23 апреля 2018г.

В окончательной редакции 27 ноября 2018 r.

Принято к публикации 29 ноября 2018г.

\begin{abstract}
Проведен сравнительный анализ механизмов лазерного возбуждения крутильных колебаний волоконных микросветоводов, который показал, что эффект Садовского, проявляющийся в поляризационно-анизотропных микросветоводах, благодаря характерным особенностям представляет особый интерес. Оценки эффективности данного механизма возбуждения колебаний и поляризационной модуляции в предложенных типах кварцевых анизотропных микросветоводов указывают на возможности создания новых типов нелинейных поляризационных элементов, волоконных поляризационных устройств, управляемых оптическим излучением, и чувствительных элементов резонансных волоконно-оптических датчиков.
\end{abstract}

DOI: 10.21883/PJTF.2019.04.47341.17353

Лазерное возбуждение крутильных колебаний волоконных микросветоводов (MF) [1] открывает новые возможности для управления состоянием поляризации излучения в MF. Это позволяет создавать новые типы нелинейных поляризационных элементов с низким порогом нелинейности и чувствительных элементов резонансных волоконно-оптических датчиков (ВОД), использующих зависимость резонансной частоты крутильных колебаний MF от внешних воздействий и параметров окружающей среды. Нелинейные поляризационные элементы представляют интерес в качестве внутрирезонаторных пассивных поляризационных модуляторов в лазерах. Резонансные ВОД на основе MF, не содержащие электрических цепей в зоне измерения, могут служить основой для разработки новых уникальных методов исследований механических (вязкоупругих) свойств биологических объектов на клеточном уровне [2]; прецизионных систем дистанционного контроля параметров взрывоопасных сред, перемещений и деформаций тел, находящихся в условиях сильных электромагнитных помех.

Механизмы лазерного возбуждения крутильных колебаний MF могут быть связаны с поглощением или рассеянием циркулярно поляризованного излучения, действием элктрического поля световой волны на поверхностные нескомпенсированные электрические заряды [3], передачей веществу орбитального или „спинового“ (эффект Садовского) углового момента оптического излучения. В нерегулярных, сложных MF крутильные колебаний могут возбуждаться также за счет параметрических эффектов (фототермического, электрострикции, фотопьезоэлектрического). Механизмы возбуждения колебаний существенно определяют (ограничивают) функциональные возможности, условия работы, области применения и методы создания устройств на основе
MF. Это обусловливает актуальность сравнительного анализа указанных механизмов, выявления оптимального (с учетом тех или иных требований) и оценки его эффективноти. Ограничиваясь рассмотрением одномодовых (в области спектра $\lambda \simeq 1.5 \mu \mathrm{m}$ ) кварцевых $\mathrm{MF}$, заметим, что орбитальный угловой момент излучения в них равен нулю и не создает крутильного момента. B таких MF при интенсивности излучения ниже порога оптической нелинейности можно пренебречь также и параметрическими эффектами (электрострикцией в поле световой волны).

Механизмы возбуждения, связанные с рассеянием поляризованного излучения и действием электрического поля световой волны на случайные поверхностные заряды, зависят от ряда факторов (распределения центров рассеяния, дефектов поверхности и др.), имеющих невоспроизводимый, случайный характер. Вследствие этого они зависят от технологических особенностей изготовления MF, внешних воздействий на $\mathrm{MF}$, условий работы, что сильно усложняет создание устройств с прогнозируемыми характеристиками, ограничивает их функциональные возможности.

Существенный недостаток механизма, основанного на поглощении поляризованного по кругу излучения, заключается в том, что неизбежные флуктуации мощности возбуждающего излучения приводят из-за термо(-оптических, -упругих, -механических) эффектов к „дрейфу“ параметров МF. Это, по существу, исключает возможность создания прецизионных устройств с высокостабильными характеристиками. Например, нестабильность резонансной частоты крутильных колебаний MF из-за нагрева возбуждающим излучением практически нивелирует преимущества частотного метода измерений. Кроме того, в области спектра $\lambda \simeq 1.5-1.6 \mu \mathrm{m}$, представляющей особый интерес, поглощение и рассея- 


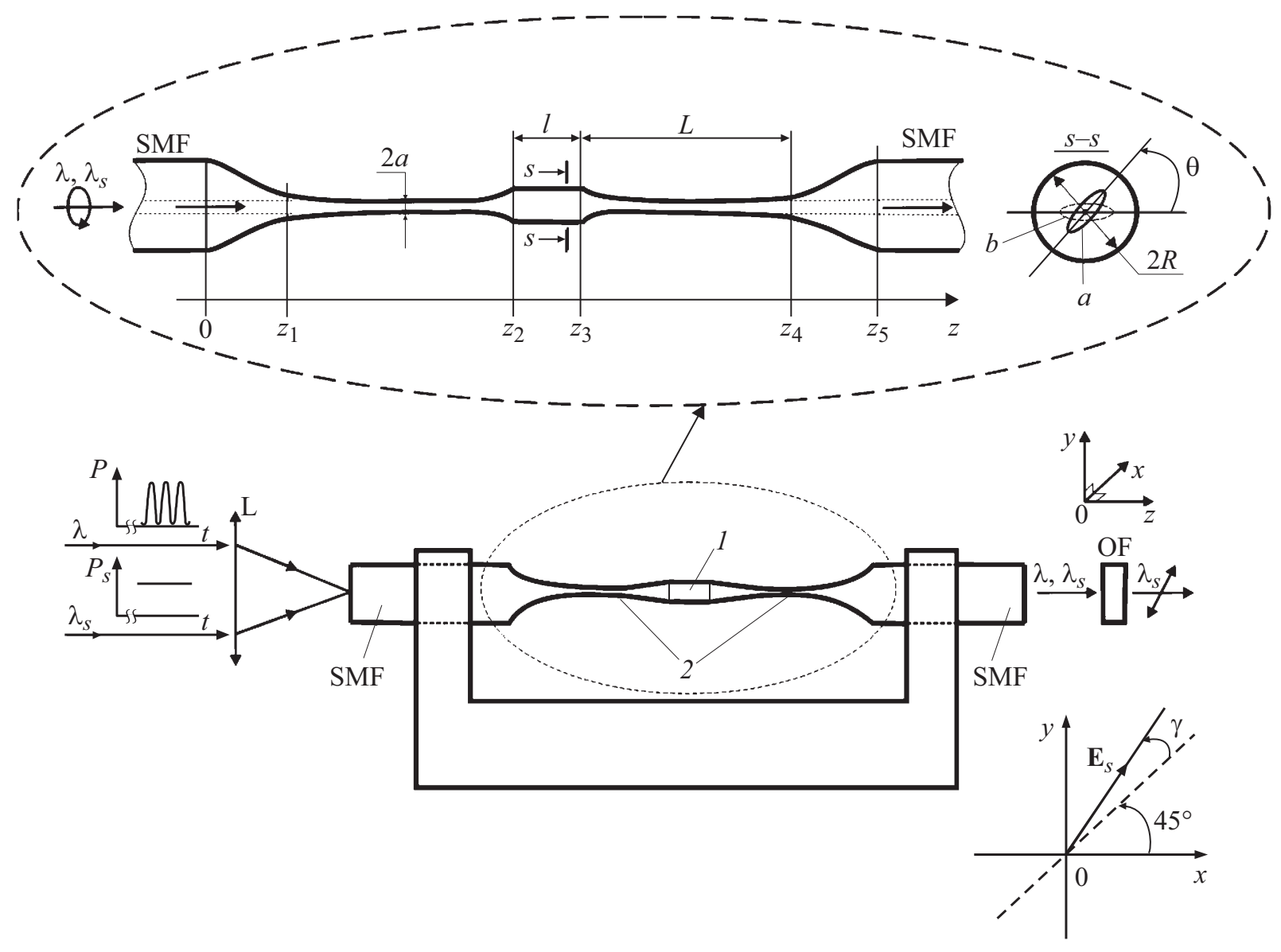

Схема волоконного поляризационно-анизотропного микросветовода. 1 - сегмент анизотропного микросветовода $\left(z_{2}, z_{3}\right) ; 2-$ волоконные биконические перетяжки $\left\{\left(0, z_{2}\right),\left(z_{3}, z_{5}\right)\right\} ;(s-s)-$ схема сечения анизотропного сегмента: $a-$ положение сердцевины анизотропного сегмента при крутильных колебаниях, $b-$ в состоянии равновесия (штриховая линия); ОF оптический фильтр; L - собирающая линза, SMF - одномодовый изотропный волоконный световод.

ние излучения в кварцевом световоде минимальные, что снижает эффективность соответствующих механизмов.

MF (как и всякий волоконный световод) в действительности всегда обладает некоторым двулучепреломлением (хотя бы „остаточным“), которое благодаря эффекту Садовского [4] приводит к возникновению в MF крутящего момента, не связанного с поглощением излучения и не зависящего от особенностей структуры анизотропного MF. Таким образом, с точки зрения возбуждения крутильных колебаний MF и создания на их основе прецизионных, высокостабильных устройств эффект Садовского представляет особый интерес. Оценки эффективности этого механизма получены с помощью упрощенной модели взаимодействия поляризационноанизотропного микросветовода (РАMF) с лазерным излучением, распространяющимся в PAMF (см. рисунок). Предложенный РАMF представляет собой составную волоконную структуру, включающую „изотропные“ волоконные биконические перетяжки и сегмент $\left(z_{2}, z_{3}\right)$ анизотропного микросветовода между ними. Указанная волоконная структура в отношении пространственного распределения двулучепреломления представляет собой упрощенную модель реальных MF, в которых максимум двулучепреломления достигается в их центре. Указанные PAMF представляют значительный самостоятельный интерес в качестве возбуждаемого светом крутильного маятника - нового класса чувствительных элементов резонансных ВОД. Заметим, что как составные части волоконной структуры, так и PAMF в целом могут быть изготовлены полностью в рамках существующих технологий волоконной оптики [5-7], обеспечивающих достаточно низкие полные потери в PAMF (не более $0.5 \mathrm{~dB})$ и позволяющих варьировать параметры PAMF $(R, l, I)$ в широких пределах.

Лазерное возбуждение крутильных колебаний в PAMF рассмотрено в рамках модели упругого неоднородного стержня с внешним распределенным крутящим моментом [8], обусловленным в данном случае лазерным излучением в PAMF:

$$
j(z) \frac{\partial^{2} \theta}{\partial t^{2}}-\frac{\partial}{\partial z}\left[G(z) I(z) \frac{\partial \theta}{\partial z}\right]=M(z, t),
$$

где $J(z)$ - погонный момент инерции элемента РАМF (с координатой $z) ; \theta(z, t)$ - угловое отклонение (пово- 
рот) из положения равновесия сечения РАMF с координатой $z$ в момент времени $t ; G(z), I(z)=\pi a^{4}(z) / 4-$ соответственно модуль сдвига и момент поперечного сечения $(a(z)$ - радиус сечения); $M(z, t)$ - погонная плотность крутящего момента, обусловленного эффектом Садовского [4]. Полагаем, что излучение на входе PAMF является циркулярно поляризованным, что не ограничивает общности рассмотрения, так как произвольное состояние поляризации можно представить в виде суперпозиции циркулярных компонент с противоположной ориентацией. Предполагая колебания РАМF малыми, считаем, что „исходное“ двулучепреломление в анизотропном сегменте $\left(z_{2}, z_{3}\right)$ значительно сильнее наведенного, обусловленного деформацией кручения. Это существенно упрощает расчет крутящего момента в анизотропном микросветоводе в условиях крутильных колебаний и позволяет рассматривать анизотропный сегмент в качестве фазовой пластины с неизменными характеристиками (приближение „твердого тела“). В частности, фазовой пластине в четверть волны $(\lambda / 4)$, переводящей круговую поляризацию в линейную, соответствует длина сегмента $l_{\lambda / 4}=\lambda / 4\left(n_{x}-n_{y}\right)$, где $n_{x}\left(n_{y}\right)$ - показатели преломления для „медленной“ („быстрой“) осей анизотропии сегмента. Например, в кварцевых РАМF с параметрами $n_{x, y} \simeq 1.46, \quad\left(n_{x}-n_{y}\right) \simeq 10^{-4}-10^{-3}$ $(\lambda=1.5 \mu \mathrm{m})$ [9] имеем $l_{\lambda / 4}=0.4-4 \mathrm{~mm}$. Радиус биконических перетяжек определяется условием одномодовости: $a \lesssim 0.38 \lambda\left(n_{x, y}^{2}-1\right)^{-1 / 2}$. Длина перетяжки $(L)$ определяется исходя из заданного диапазона собственных частот $(\omega)$ крутильных колебаний РАMF (крутильной жесткости перетяжек $\left.C \approx \pi a^{4} G / L\right)$.

При колебаниях РАMF деформация кручения в биконических перетяжках приводит к возникновению в них наведенного двулучепреломления. Это приводит к различию состояний поляризации на входе $\mathrm{PAMF}(z=0)$ и анизотропного сегмента $\left(z=z_{2}\right)$. Как известно [10], скручивание кварцевого волоконного световода (на угол $\theta)$ приводит к повороту плоскости поляризации в противоположном направлении на значительно меньший угол: $(-0.07) \theta$. Таким образом, в условиях малых колебаний $\theta \ll 1$ при расчете крутящего момента в анизотропном сегменте искажением круговой поляризации излучения на его входе можно пренебречь (при $\theta \approx 0.1 \mathrm{rad}$ погрешность не превышает 5\%). С близкой точностью $(6 \%)$ можно пренебречь потерями мощности излучения в PAMF и с точностью $3 \%$ крутящим моментом в биконических перетяжках, возникающим из-за наведенной анизотропии. В итоге с достаточной для оценок точностью функция распределения погонной плотности крутящего момента в PAMF имеет вид

$M(z, t)= \begin{cases}0, & 0<z \leqslant z_{2}, \\ \frac{1}{2 c}\left(n_{x}^{2}-n_{y}^{2}\right) p(t) \sin \left(\frac{2 \pi\left(n_{x}-n_{y}\right) z}{\lambda}\right), & z_{2}<z \leqslant z_{3}, \\ 0, & z_{3}<z \leqslant z_{5},\end{cases}$ где $p(t)$ - мощность лазерного излучения в PAMF, $c-$ скорость света в вакууме.

PAMF характеризуется широким набором мод собственных крутильных колебаний. С точки зрения создания резонансных ВОД и эффективных поляризационных модуляторов особый интерес представляют резонансные колебания на собственной частоте основной моды крутильных колебаний. Поиск приближенного решения уравнения (1) в виде $\theta(z, t) \approx \theta(t) \theta(z)$, где $\theta(z)-$ форма основной моды собственных крутильных колебаний $\left(\theta\left(z_{2}+l / 2\right)=1\right)$, с учетом (2) и соотношений $R \gg a$, $L \gg l$, характерных для РАMF, приводит к уравнению вынужденных крутильных колебаний анизотропного сегмента

$$
\ddot{\theta}+\frac{\omega}{Q} \dot{\theta}+\omega^{2} \theta=K p(t),
$$

где $K=\left(n_{x}+n_{y}\right) \lambda / 4 \pi J_{0} c ; \omega=\left(C / J_{0}\right)^{1 / 2} ; J_{0} \approx \pi R^{4} l \rho / 4$ $(\rho-$ плотность материала). Дополнительное слагаемое $\omega \dot{\theta} / Q$ в (3) учитывает затухание колебаний, связанное с вязкостью окружающей среды и внутренним сопротивлением в материале $\mathrm{PAMF}(Q-$ механическая добротность). При гармонической модуляции мощности $p(t)=p_{0}(1+\cos (\Omega t)), \Omega \approx \omega$, решение уравнения (3) имеет вид

$$
\theta(t)=\bar{\theta}+\theta_{0}(\Omega) \cos (\Omega t+\varphi)
$$

где $\bar{\theta}=K p_{0} / \omega^{2}-$ статический угол поворота, $\theta_{0}(\Omega)$ - амплитудно-частотная характеристика вынужденных крутильных колебаний с амплитудой в резонансе: $\theta_{0}(\Omega \approx \omega) \approx Q \bar{\theta}$. Например, при $p_{0}=1 \mathrm{~mW}$ в PAMF с параметрами $R=1.5 \mu \mathrm{m}, l_{\lambda / 4}=1 \mathrm{~mm}$, $a=0.3 \mu \mathrm{m}, L=5 \mathrm{~mm}, f=23 \mathrm{kHz}, J_{0}=8 \cdot 10^{-24} \mathrm{~kg} \cdot \mathrm{m}^{2}$, $C=1.5 \cdot 10^{-13} \mathrm{H} \cdot \mathrm{m}, Q=2 \cdot 10^{4}[3]$ амплитуда резонансных колебаний составляет $\theta_{0}(\omega) \approx 0.14 \mathrm{rad}$, что почти на три порядка превышает уровень флуктуационных („тепловых“) колебаний при температуре $T=300 \mathrm{~K}$ : $\langle\theta\rangle_{f l} \simeq(2 k T / C)^{1 / 2} \approx 2.4 \cdot 10^{-4} \mathrm{rad} \quad(k-$ постоянная Больцмана).

Анализ поляризационного состояния излучения в PAMF, учитывающий наведенную анизотропию в перетяжках $\left\{\left(0, z_{2}\right),\left(z_{3}, z_{5}\right)\right\}$, может быть выполнен с помощью метода матриц Джонса. Он показывает, что при малых, низкочастотных крутильных колебаниях $\left(\theta_{0} \ll 1\right.$, $f \ll c / 2 L)$ пробное, поляризованное по кругу непрерывное излучение $\left(p_{s} \ll p_{0}, \lambda_{s} \approx \lambda\right)$ с помощью PAMF преобразуется в поляризационно-модулированное (см. рисунок) с плоскостью линейной поляризации, качающейся вокруг оси РАMF на угол $\gamma(t)=\gamma_{0}(\Omega) \cos (\Omega t+\varphi)$, где амплитуда колебаний плоскости поляризации $\gamma_{0}(\Omega) \approx \theta_{0}(\Omega), \varphi \approx \operatorname{arctg}\left(\Omega^{2} / Q\left(\omega^{2}-\Omega^{2}\right)\right)$.

Таким образом, в работе показано, что эффект Садовского является достаточно эффективным механизмом лазерного возбуждения крутильных колебаний кварцевых анизотропных микросветоводов, который с учетом его характерных особенностей открывает возможности создания высокоточных волоконных поляризационных 
устройств и резонансных ВОД в полностью волоконном исполнении, что расширяет области их применения.

\section{Список литературы}

[1] Fenton E., Khan A., Patterson B., Solano P., Rolston S.L., Orozco L.A., Fatemi F.K. Torsional modes of a nanofiber: polarimetric excitation and read out // Proc.of Frontier in optics 2016. N.Y.: Rochester, 2016. P. FTu3I.6.

[2] Gardel M., Oakes P. Measuring cell mechanics. Morgan \& Claypool Publ., 2015. 76 p.

[3] Wuttke C., Cole G.D., Rauschenbeutel A. // Phys. Rev. A. 2013. V. 88. P. 061801R.

[4] Валитов Р.А., Хижняк Н.А., Жилков В.С. Пондеромоторное действие электромагнитного поля (теория и приложения) / Под. ред. Р.А. Валитова. М.: Сов. радио, 1975. $232 \mathrm{c}$

[5] Jung Y., Brambilla G., Richardson D.J. // OECC 2010 Technical Digest. IEEE, 2010. P. 790-791.

[6] Chang C.D., Chuo S.M., Wang L.A. // Proc. SPIE. 2011. V. 7753. P. 775367.

[7] Yarin A., Pourdeyhimi B., Ramakrishna S. Fundamentals and applications of micro- and nanofibers.Cambridge University Press, 2013. https://doi.org/10.1017/CBO9781107446830

[8] Бабаков И.М. Теория колебаний. М.: Наука, 1968. 560 с.

[9] Андреев А.Г., Буреев С.В., Ероньян М.А. // Опт. журн. 2012. T. 79. № 9. C. 107-109.

[10] Budinski V., Donlagic D. // Sensors. 2017. V. 17. N 3. P. 443 (1-29). 